\title{
A Global Approach with Cutoff Exponential Function, Mathematically Well Defined at the Outset, for Calculating the Casimir Energy: The Example of Scalar Field
}

\author{
M. S. R. Miltão and Franz A. Farias \\ Departamento de Física-UEFS, 44036-900 Feira de Santana, BA, Brazil \\ Correspondence should be addressed to M.S.R. Miltão, miltaaao@ig.com.br
}

Received 9 October 2010; Accepted 6 December 2010

Academic Editor: Ira Rothstein

Copyright (C) 2010 M.S.R. Miltão and F. A. Farias. This is an open access article distributed under the Creative Commons Attribution License, which permits unrestricted use, distribution, and reproduction in any medium, provided the original work is properly cited.

A global approach with cutoff exponential functions is used to obtain the Casimir energy of a massless scalar field in the presence of a spherical shell. The proposed method, mathematically well defined at the outset, makes use of two regulators, one of them to make the sum of the orders of Bessel functions finite and the other to regularize the integral involving the zeros of Bessel function. This procedure ensures a consistent mathematical handling in the calculations of the Casimir energy and allows a major comprehension on the regularization process when nontrivial symmetries are under consideration. In particular, we determine the Casimir energy of a scalar field, showing all kinds of divergences. We consider separately the contributions of the inner and outer regions of a spherical shell and show that the results obtained are in agreement with those known in the literature, and this gives a confirmation for the consistence of the proposed approach. The choice of the scalar field was due to its simplicity in terms of physical quantity spin.

\section{Introduction}

The relevance of the Casimir effect has increased over the decades since the seminal paper (1948) [1] by the Dutch Physicist Hendrik Casimir. This effect concerns to the appearance of an attractive force between two plates when they are placed close to each other. Casimir was the first to predict and explain the effect as a change in vacuum quantum fluctuations of the electromagnetic field. 
Nowadays, the Casimir effect has been applied to a variety of quantum fields and geometries and it has gained a wider understanding as the effect which comes from the fluctuations of the zero point energy of a relativistic quantum field due to changes in its base manifold. This interpretation can be confirmed when we see the large range where the Casimir effect has been applied: the study of gauge fields with BRS symmetry [2], in the Higgs fields [3], in supersymmetric fields [4], in supergravity theory [5, 6], in superstrings [7], in the Maxwell-Chern-Simons fields [8], in relativistic strings [9], in M-theory [10], in cosmology [11], and in noncommutative spacetimes [12], among other subjects in the literature [13, 14], the review articles [15-17], and textbooks [18-23].

In this present work, the meaning of base manifold is that the confinement that the field is subjected is due to the presence of a sphere, where the boundary conditions take place. The point we aim to emphasize is that once the calculation of the Casimir effect involves dealing with infinite quantities, we need to use a regularization procedure appropriately defined. Many different regularization methods have been proposed and we can quote some of them: the summation mode method-using the general cutoff function [1], exponential cutoff function [24, 25], Green function [26-30], Green function through multiple scattering [31], exponential function and cutoff parameter [32, 33], zeta function [34-40], Abel-Plana formula [21, 41], or point-splitting [42-44]; the Green function methodusing the point-splitting [45-48], Schwinger's source theory [49-51], or zeta function [52]; the statistical approach method-using the path integral formalism [53], or Green function [54]; as some examples among others. These methods are distinguished by the approach used to carry out the calculations of the Casimir energy, and it is clear that the physical result must be independent from the regulators or the method employed for them. But the literature has shown that the results found there exhibit a divergence among them.

In a general way, the methods used to obtain the Casimir effect lie on one of the two categories: a local procedure or a global one. With a local procedure, we mean one that the expression for the change of the vacuum energy is explicitly dependent on the variables of the base manifold, and only in the final step of calculations the integration over these variables is carried out. On the other hand, in a global one, we start with an expression for the vacuum energy where there is no space-time variables present as they already were integrated.

In the present work, we pretend detailing a global approach $[55,56]$ for the calculation of Casimir energy. In this method, mathematically well defined at the outset, we propose the use of two regulators into the cutoff exponential function, and we demonstrate that this regularization approach is one appropriate for the calculation of Casimir effect in the case of nontrivial symmetries, in particular a spherical symmetry.

With the use of scalar field, we can avoid the inherent complications brought by the vector nature of the electromagnetic field, and due to its simple structure, the scalar field usually becomes an effective tool to be used in the investigation of field proprieties as in these examples: in the dynamical Casimir effect [57], in the Casimir effect at finite temperature $[58,59]$, and in the Casimir effect on a presence of a gravitational field [60, 61], among others [62-64].

The paper is organized as follows: we detail in Section 2 the method to be used and why we need two cutoff parameters to obtain an regularized expression for the Casimir energy, which is the starting point for a consistent mathematical handling. Section 3 exhibits the calculations for the contributions of the inner and the outer regions of the spherical shell. We analyze in Section 4 the results and compare them with those ones in the literature and make some considerations. 


\section{The Global Procedure Proposed with Two Parameters}

The starting point is the expression for the Casimir energy defined as the difference between the vacuum energy under a given boundary condition and the reference vacuum energy. When we consider a scalar field in the presence of a spherical shell, this vacuum energy is

$$
E_{0}=\sum_{n=1}^{\infty} \sum_{j=0}^{\infty} \sum_{m=-j}^{j} \sum_{\tau} \frac{1}{2} \hbar \omega_{j n}^{\tau}
$$

where $\omega_{j n}^{\tau}$ are the mode frequencies. They are obtained when the boundary conditions are imposed on the field. In the absence of boundary conditions, the frequencies take some values which let us designate as $\omega_{j n}^{\tau(\mathrm{ref})}$ and these lead to the vacuum reference energy

$$
E^{(\mathrm{ref})}=\sum_{n=1}^{\infty} \sum_{j=0}^{\infty} \sum_{m=-j}^{j} \sum_{\tau} \frac{1}{2} \hbar \omega_{j n}^{\tau(\mathrm{ref})},
$$

so the Casimir energy is $\mathcal{\varepsilon}=E_{0}-E^{(\mathrm{ref})}$. The boundary conditions due to a spherical shell with radius $a$ are

$$
\begin{gathered}
k a j_{j}(k a)=0, \quad \text { for } r=a-0, \\
A_{j} k a j_{j}(k a)+B_{j} k a n_{j}(k a)=0, \quad \text { for } r=a+0 .
\end{gathered}
$$

The Casimir energy will be calculated by using the mode summation and the argument theorem (also known as argument principle [65-67]). This theorem gives the summation of zeros and poles of an analytic function as a contour integral. This contour is a curve that encompasses the interior region of the complex plane which contains the zeros and poles [65-67]. In our case, we are interested in the root functions which match the conditions (2.3). So, the following equations are appropriate as root functions

$$
\begin{aligned}
& f_{j}^{1}(a z)=a z j_{j}(a z), \\
& f_{j}^{2}(a z)=\cos \delta_{j}(z)\left[a z j_{j}(a z)+\tan \delta_{j}(z) a z n_{j}(a z)\right],
\end{aligned}
$$

where

$$
z=k\left(=\frac{\omega}{c}\right), \quad \delta_{j}(z)=z R-\frac{j \pi}{2} .
$$

When we apply the argument theorem and carry out some handling, we get

$$
\sum_{n=1}^{g} \omega_{j n}^{\tau}=\frac{c}{2 \pi i} \oint_{C} d z z \frac{d}{d z} \log f_{j}^{\tau}(a z)
$$




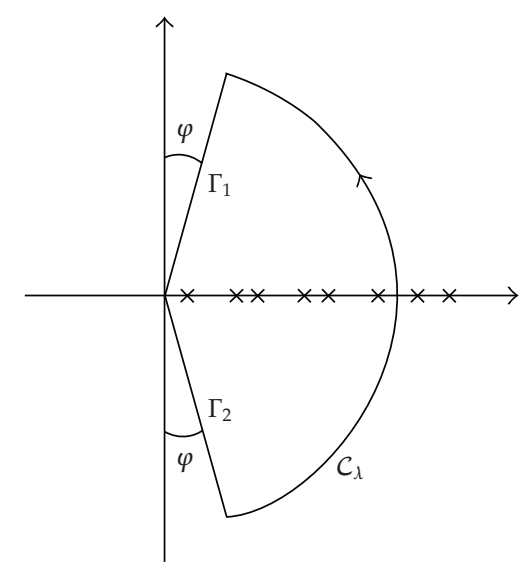

Figure 1: The path of integration in the complex plane.
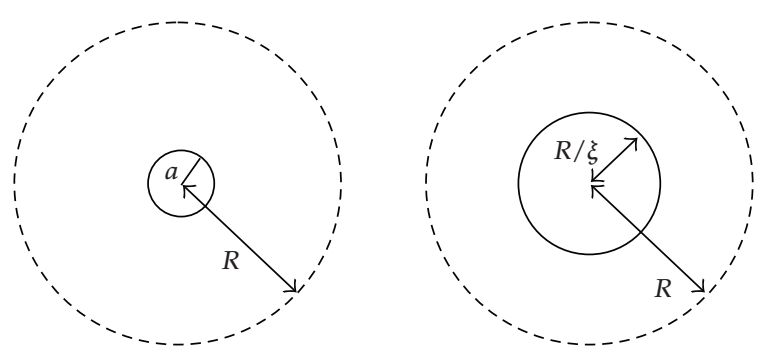

Figure 2: A sketch of the subtraction process which takes place on the regularization of the Casimir effect of a spherical shell.

In the above equation, the argument for logarithm must involve the product of all root functions. The contour to be taken on the calculations is given by [68] according to Figure 1.

The subtraction process (renormalization), defined by $\mathcal{E}$, can be schematically represented as in Figure 2.

The vacuum energy (2.1) which takes into account the boundary conditions can be used to obtain the reference energy in (2.2). This is done when we take the limit for the radius $a$ going to infinity. This procedure is sensible, but it already has been made clear by Boyer $[69,70]$. After all, we obtain for the Casimir energy

$$
\begin{aligned}
\mathcal{E}= & \sum_{n=1}^{\infty} \sum_{j=0}^{\infty} \sum_{m=-j}^{j} \sum_{\tau=1}^{4} \frac{1}{2} \hbar\left(\omega_{j n}^{\tau}-\omega_{j n}^{\tau(\mathrm{ref})}\right) \\
= & \lim _{\sigma \rightarrow 0, \epsilon \rightarrow 0, R \rightarrow \infty, \xi \rightarrow 1} \frac{\hbar c}{2 \pi i} \sum_{j=0}^{\infty} v \exp (-\epsilon v) \oint_{C} d z z \exp (-\sigma z) \\
& \times \frac{d}{d z}\left\{\log \left[f_{j}^{(1)}(a z) f_{j}^{(2)}(a z)\right]-\log \left[f_{j}^{(1)(\mathrm{ref})}\left(\frac{R}{\xi} z\right) f_{j}^{(2)(\mathrm{ref})}\left(\frac{R}{\xi} z\right)\right]\right\},
\end{aligned}
$$


where $v=j+1 / 2$. We can see from above that two exponential functions were used, one of them is the function under the integral sign, $\exp (-\sigma z),(\sigma>0)$, that stems from the argument theorem and the other is the function $\exp (-\epsilon v),(\epsilon>0)$, under summation sign on $j=v-$ $(1 / 2)$. Now, group together these two developments, and (2.7) may be rewritten as

$$
\begin{aligned}
& \mathcal{E}=-\frac{\hbar c}{\pi} \Re \sum_{j=0}^{\infty} \mathcal{v}^{2} \exp (-\epsilon \mathcal{v}) \int_{0}^{\infty} \exp (-i \varphi) d z \exp (-i \sigma v z) z \frac{d}{d z}\left\{\log \left[I_{\mathcal{v}}(v a z)\right]+\log \left[K_{v}(v a z)\right]\right\} \\
& -E^{(\mathrm{ref})} \text {, }
\end{aligned}
$$

where the limits for $R$ and $\xi$ have been taken into account. The other limits will be taken in an appropriate moment after the cancelation of possible remaining divergences.

\section{Casimir Effect of a Spherical Shell: The Case of a Scalar Field}

We now rewrite (2.8) in a more appropriate way, so that the contributions can be separated by regions as $\varepsilon=\varepsilon_{I}+\varepsilon_{O}$, where

$$
\begin{aligned}
\mathcal{E}_{I}= & -\frac{\hbar c}{\pi} \Re\left(\frac{1}{2}\right)^{2} \exp \left(-\epsilon \frac{1}{2}\right) \int_{0}^{\infty \exp (-i \varphi)} d z z \exp \left(-i \sigma \frac{1}{2} z\right) \frac{d}{d z}\left\{\log \left[I_{1 / 2}\left(\frac{1}{2} a z\right)\right]\right\} \\
& -\frac{\hbar c}{\pi} \Re \sum_{j=1}^{\infty} v^{2} \exp (-\epsilon \mathcal{v}) \int_{0}^{\infty \exp (-i \varphi)} d z z \exp (-i \sigma v z) \frac{d}{d z}\left\{\log \left[I_{v}(v a z)\right]\right\}-E_{I}^{\text {ref }}
\end{aligned}
$$

is the contribution due to the internal modes and

$$
\begin{aligned}
\mathcal{E}_{O}= & -\frac{\hbar c}{\pi} \Re\left(\frac{1}{2}\right)^{2} \exp \left(-\epsilon \frac{1}{2}\right) \int_{0}^{\infty \exp (-i \varphi)} d z z \exp \left(-i \sigma \frac{1}{2} z\right) \frac{d}{d z}\left\{\log \left[K_{1 / 2}\left(\frac{1}{2} a z\right)\right]\right\} \\
& -\frac{\hbar c}{\pi} \Re \sum_{j=1}^{\infty} v^{2} \exp (-\epsilon \mathcal{v}) \int_{0}^{\infty \exp (-i \varphi)} d z z \exp (-i \sigma v z) \frac{d}{d z}\left\{\log \left[K_{v}(v a z)\right]\right\}-E_{O}^{\text {ref }}
\end{aligned}
$$

is the contribution due to the external modes. As it can be observed, the above contributions were written in such a way that the term for $j=0$ was detached from the summation on $j$. This has been done to the effect of making explicit the term on which we will focus attention as well as taking into account some developments already accomplished [55].

\subsection{Internal Mode}

Now, we proceed with the calculations of (3.1), and the first step is to take the Debye expansion for the Bessel functions up to order $\mathcal{O}\left(v^{-4}\right)[71,72]$. The Debye expansion gives 
accurate results when we consider large order of $v=j+1 / 2$ and larger arguments and that also makes an analytical treatment possible for the resulting expressions. So, we have

$$
\varepsilon_{I}=E_{I}-E_{I}^{(\mathrm{ref})}
$$

where $E_{I}=E_{I, 0}+E_{I, 1}+E_{I, 2}+E_{I, 3}+E_{I, 4}$ and the terms $E_{I, n}$ are given by

$$
\begin{aligned}
& E_{I, 0}=-\frac{\hbar c}{2 \pi} \Re \exp \left(-\epsilon \frac{1}{2}\right) \exp (-i \varphi) \int_{0}^{\infty} d \rho \exp (-i \sigma \rho \exp (-i \varphi)) \\
& \times\left[-\frac{1}{2}+\exp (-i \varphi) a \rho \operatorname{coth}(a \rho \exp (-i \varphi))\right], \\
& E_{I, 1}=\frac{\hbar c}{\pi a} \sum_{j=1}^{\infty} v^{2} \int_{0}^{\infty} d \rho\left\{\log \left[\varrho_{I}(v, \rho)\right]-\sum_{k=1}^{4} \frac{u_{(I, k)}(t)}{v^{k}}\right\} \text {, } \\
& E_{I, 2}=-\frac{\hbar c}{\pi} \Re \sum_{k=1}^{4} \sum_{j=1}^{\infty} v^{2-k} \exp (-\epsilon v) \int_{0}^{\infty \exp (-i \varphi)} d z \exp (-i \sigma v z) z \frac{d}{d z} \boldsymbol{U}_{(I, k)}(t), \\
& E_{I, 3}=\frac{\hbar c}{2 \pi} \Re \sum_{j=1}^{\infty} v^{2} \exp (-\epsilon \mathcal{v}) \int_{0}^{\infty} \exp (-i \varphi)^{2} d z \exp (-i \sigma v z) \frac{a^{2} z^{2}}{1+a^{2} z^{2}} \\
& E_{I, 4}=-\frac{\hbar c}{\pi} \Re \sum_{j=1}^{\infty} v^{3} \exp (-\epsilon \mathcal{V}) \int_{0}^{\infty \exp (-i \varphi)} d z \exp (-i \sigma v z) \sqrt{1+a^{2} z^{2}}
\end{aligned}
$$

with the definitions [37]

$$
\begin{aligned}
& \supset_{I}(v, \rho)=\sqrt{2 \pi v}\left(1+\rho^{2}\right)^{1 / 4} \exp (-v \eta) I_{v}(v \rho), \\
& u_{(I, 1)}(t)=\frac{t}{8}-\frac{5 t^{3}}{24} \\
& \varkappa_{(I, 2)}(t)=\frac{t^{2}}{16}-\frac{3 t^{4}}{8}+\frac{5 t^{6}}{16} \\
& u_{(I, 3)}(t)=\frac{25 t^{3}}{384}-\frac{531 t^{5}}{640}+\frac{221 t^{7}}{128}-\frac{1105 t^{9}}{1152} \\
& u_{(I, 4)}(t)=\frac{13 t^{4}}{128}-\frac{71 t^{6}}{32}+\frac{531 t^{8}}{64}-\frac{339 t^{10}}{32}+\frac{565 t^{12}}{128} .
\end{aligned}
$$

The contributions (3.7) and (3.8) compound the zero-order terms of the Debye expansion. The contribution (3.5) stems from small values of the angular momentum $j$, and its value was already determined by [37]

$$
E_{I, 1}=0.00024 \frac{\hbar c}{\pi a}
$$


The contributions (3.6) to (3.8) are calculated taking into account the Euler-Maclaurin formula with remainder [73-75], and these were calculated by [55]

$$
\begin{aligned}
& E_{I, 2}=\frac{\hbar c}{\pi} \Re\left\{\frac{8099}{63839} \frac{1}{a}+\frac{7}{24} \frac{a}{\sigma^{2}}+\frac{11}{192} \frac{1}{a} \log \left(\frac{\sigma}{a}\right)+\frac{229}{40320} \frac{1}{a} \log (\epsilon)+i \frac{7801}{86684} \frac{1}{a}\right\}, \\
& E_{I, 3}=\frac{\hbar c}{\pi} \Re\left\{\frac{52529}{267528} \frac{1}{a}+i\left[-\frac{1}{3} \frac{1}{\sigma}-\frac{3}{4} \frac{1}{\sigma \epsilon}-\frac{1}{2} \frac{1}{\sigma \epsilon^{2}}\right]\right\}, \\
& E_{I, 4}=\frac{\hbar c}{\pi} \Re\left\{\frac{7375}{85696} \frac{1}{a}-\frac{11}{24} \frac{a}{\sigma^{2}}+2 \frac{a^{3}}{\sigma^{4}}-\frac{127}{1920} \frac{1}{a} \log \left(\frac{\sigma}{a}\right)-i \frac{2197}{21145} \frac{1}{a}\right\} .
\end{aligned}
$$

Collecting the terms (3.14), (3.15), (3.16), and (3.17), we get

$$
E_{I_{\text {partial }}}=0.4095155894 \frac{\hbar c}{\pi a}+\frac{\hbar c}{\pi}\left[-\frac{1}{6} \frac{a}{\sigma^{2}}+2 \frac{a^{3}}{\sigma^{4}}-\frac{17}{1920} \frac{1}{a} \log \left(\frac{\sigma}{a}\right)+\frac{229}{40320} \frac{1}{a} \log (\epsilon)\right] .
$$

For (3.4), corresponding to $j=0$, we obtain

$$
E_{I, 0}=\frac{\hbar c}{\pi}\left[-\frac{1}{24} \frac{\pi^{2}}{a}+\frac{1}{2} \frac{a}{\sigma^{2}}\right]
$$

So, the energy of a scalar field considering a spherical configuration due the internal modes is

$$
\begin{aligned}
E_{I} & =E_{I_{\text {partial }}}+E_{I, 0} \\
& =-\frac{\hbar c}{\pi a} 0.0017179275+\frac{\hbar c}{\pi}\left[\frac{1}{3} \frac{a}{\sigma^{2}}+2 \frac{a^{3}}{\sigma^{4}}-\frac{17}{1920} \frac{1}{a} \log \left(\frac{\sigma}{a}\right)+\frac{229}{40320} \frac{1}{a} \log (\epsilon)\right] .
\end{aligned}
$$

The expression (3.20) shows in an undoubted way the need for a second regularized exponential function, $\exp (-\epsilon \mathcal{V})$, to make a consistent mathematical handling of the divergences possible. Both divergences, the logarithm in (3.15) and the polynomial in (3.16), stem from the summation on $j$. This type of divergence was already observed in [76], but only with the procedure established here this discard turns to be completely justified as an appropriate regularization allows the real part of (3.16) to be taken in an unambiguous way.

\subsection{External Mode}

The contribution of the external modes comes by (3.2). We proceed with the calculations in an analogous way to that of the previous subsection. So,

$$
\varepsilon_{O}=E_{O}-E_{O}^{(\mathrm{ref})}
$$


where $E_{O}=E_{O, 0}+E_{O, 1}+E_{O, 2}+E_{O, 3}+E_{O, 4}$ and

$$
\begin{aligned}
& E_{O, 0}=-\frac{\hbar c}{2 \pi} \Re \exp \left(-\epsilon \frac{1}{2}\right) \exp (-i \varphi) \int_{0}^{\infty} d \rho \exp (-i \sigma \rho \exp (-i \varphi))\left[-\frac{1}{2}-\exp (-i \varphi) a \rho\right], \\
& E_{O, 1}=\frac{\hbar c}{\pi a} \sum_{j=1}^{\infty} v^{2} \int_{0}^{\infty} d \rho\left\{\log \left[\mathcal{K}_{O}(v, \rho)\right]-\sum_{k=1}^{4} \frac{\boldsymbol{u}_{(O, k)}(t)}{v^{k}}\right\}, \\
& E_{0,2}=-\frac{\hbar c}{\pi} \Re \sum_{k=1}^{4} \sum_{j=1}^{\infty} v^{2-k} \exp (-\epsilon \mathcal{v}) \int_{0}^{\infty \exp (-i \varphi)} d z \exp (-i \sigma v z) z \frac{d}{d z} \boldsymbol{U}_{(O, k)}(t), \\
& E_{O, 3}=\frac{\hbar c}{2 \pi} \Re \sum_{j=1}^{\infty} v^{2} \exp (-\epsilon \mathcal{v}) \int_{0}^{\infty \exp (-i \varphi)} d z \exp (-i \sigma v z) \frac{a^{2} z^{2}}{1+a^{2} z^{2}} \\
& E_{O, 4}=\frac{\hbar c}{\pi} \Re \sum_{j=1}^{\infty} v^{3} \exp (-\epsilon \mathcal{v}) \int_{0}^{\infty \exp (-i \varphi)} d z \exp (-i \sigma v z) \sqrt{1+a^{2} z^{2}}
\end{aligned}
$$

with the following definitions [37]:

$$
\begin{aligned}
& \mathcal{K}_{O}(v, \rho)=\sqrt{\frac{2 v}{\pi}}\left(1+\rho^{2}\right)^{1 / 4} \exp (v \eta) K_{v}(v \rho) \\
& \mathcal{U}_{(O, 1)}(t)=-\mathcal{U}_{(I, 1)}(t) \\
& \boldsymbol{U}_{(O, 2)}(t)=\boldsymbol{U}_{(I, 2)}(t) \\
& \boldsymbol{U}_{(O, 3)}(t)=-\boldsymbol{U}_{(I, 3)}(t) \\
& \boldsymbol{U}_{(O, 4)}(t)=\boldsymbol{U}_{(I, 4)}(t)
\end{aligned}
$$

where the $\mathfrak{U}_{(O, k)}$ are given by (3.10) to (3.13), respectively. The term (3.28) was numerically determined by [37]

$$
E_{O, 1}=-0.00054 \frac{\hbar c}{\pi a}
$$

The other contributions are calculated following the analogous procedure detailed in the previous subsection:

$$
\begin{aligned}
& E_{O, 2}=\frac{\hbar c}{\pi} \Re\left\{-\frac{5821}{56688} \frac{1}{a}-\frac{7}{24} \frac{a}{\sigma^{2}}-\frac{11}{192} \frac{1}{a} \log \left(\frac{\sigma}{a}\right)-\frac{229}{40320} \frac{1}{a} \log (\epsilon)-i \frac{7801}{86684} \frac{1}{a}\right\}, \\
& E_{O, 3}=\mathcal{E}_{I, 3}, \\
& E_{O, 4}=-\mathcal{\varepsilon}_{I, 4} .
\end{aligned}
$$


After collecting the terms (3.32) and (3.33), we have

$$
E_{O_{\text {partial }}}=0.01056399145 \frac{\hbar c}{\pi a}+\frac{\hbar c}{\pi}\left[\frac{1}{6} \frac{a}{\sigma^{2}}-2 \frac{a^{3}}{\sigma^{4}}+\frac{17}{1920} \frac{1}{a} \log \left(\frac{\sigma}{a}\right)-\frac{229}{40320} \frac{1}{a} \log (\epsilon)\right] .
$$

The contribution (3.22), related to $j=0$, when we repeat the calculation gives

$$
E_{O, 0}=\frac{\hbar c}{\pi}\left[-\frac{1}{2} \frac{a}{\sigma^{2}}\right]
$$

Gathering together (3.34) and (3.35), we get the total contribution to the energy of a scalar field of a spherical configuration due the external modes

$$
\begin{aligned}
E_{O} & =E_{O_{\text {partial }}}+E_{O, 0} \\
& =\frac{\hbar c}{\pi a} 0.01056399145+\frac{\hbar c}{\pi}\left[-\frac{1}{3} \frac{a}{\sigma^{2}}-2 \frac{a^{3}}{\sigma^{4}}+\frac{17}{1920} \frac{1}{a} \log \left(\frac{\sigma}{a}\right)-\frac{229}{40320} \frac{1}{a} \log (\epsilon)\right] .
\end{aligned}
$$

Our next task is to determine the reference energy and take the regularizations as indicated by (3.3) and (3.21).

\section{The Regularized Results}

Calculating the reference energy, we get

$$
E_{ \pm}^{(\mathrm{ref})}= \pm \frac{R}{\xi} \frac{f(\epsilon)}{\sigma^{2}}
$$

where the plus sign refers to $\varepsilon_{I}$ and the minus sign to $\varepsilon_{O}$ and

$$
f(\epsilon)=\exp \left(-\frac{\epsilon}{2}\right)(3 \exp (\epsilon)-1)(\exp (\epsilon)-1)^{-2}
$$

Now, we can gather together the internal (3.20) and external (3.36) contributions, taking into account (4.1), to obtain the Casimir effect for a scalar field due to the presence of a spherical shell with radius $a$

$$
\mathcal{E}(a)=\frac{\hbar c}{a} 0.002815789609
$$

This result is free of divergences since we get an exact cancelation for the terms which depend on the cutoff parameters. Equation (4.3) is in agreement with that obtained by [37], through the zeta function method, and with that in [77], which uses the Green function formalism and the dimensional analytical extension (in this reference the starting point is the expression for the force). 


\section{Conclusions}

Our purpose in this work was to show the form and nature of each divergent term that appears in the calculation of Casimir energy and demonstrate that the method proposed is mathematically consistent and that it is in accordance with the results existing in literature.

To this end, we show explicitly, to the scalar field, the characteristic of the divergent terms calculated in (3.20), (3.36), and (4.1) as a function of the geometrical proprieties of boundary if we rewrite the divergent part of those assuming a dimensionless parameter $\varepsilon=$ $(\epsilon a)^{357 / 155}$, with $\operatorname{dim}(\epsilon)=L^{-1}$, so

$$
\mathcal{E}_{ \pm}= \pm \hbar c\left[\frac{3}{\pi^{2}} V(a) \frac{1}{\sigma^{4}}-\frac{17}{3840 \pi^{2}} S(a) \kappa^{3} \log (\sigma \varepsilon)+\frac{1}{3 \pi^{2}} S(a) \kappa \frac{1}{\sigma^{2}}-\frac{1}{4 \pi^{2}} S\left(\frac{R}{\xi}\right) \kappa S\left(\frac{R}{\xi}\right) \frac{f(\varepsilon)}{\sigma^{2}}\right],
$$

where the plus sign refers to the index $I$ while the minus sign refers to the index $O$, and $\mathcal{\kappa}=1 / a$ is the curvature. In (5.1), $V(a)$ is a volume, $S(a)$ is an area, and $\sigma$ and $\epsilon$ are cutoff parameters. As we can see, the second and fourth terms in (5.1) explain why two regulators are required to get a well-defined expression for the Casimir energy of the scalar field. This is the same case when we consider an electromagnetic field (see [55]). The result (5.1) is in agreement with [78], except for the divergence due to $\log (\epsilon)$ and due to the relative selfenergy of the spherical shell, $f(\varepsilon) / \sigma^{2}$, that does not appear there.

Our purpose in this work is to confirm that the prescription in (2.7) works well when we assume non trivial symmetries for the fields. In fact, the approach has succeed in demonstrating the cancelation of all types of divergences appearing in the expression for the Casimir energy of the scalar field. Besides, this calculation presented at this work shows the desired agreement with the results existing in the literature. Furthermore, as it has been mentioned by the authors in [79-81], a better understanding of the quantum field theory undoubtedly involves the necessity to understand these infinities.

\section{Acknowledgment}

The authors wish to thank Dr. Ludmila Oliveira H. Cavalcante (DEDU-UEFS) for valuable help with English revision.

\section{References}

[1] H. B. G. Casimir, "On the attraction between two perfectly conducting plates," Proceedings of the Royal Netherlands Academy of Arts and Sciences, vol. 51, pp. 793-795, 1984.

[2] J. Ambjørn and R. J. Hughes, "Gauge fields, BRS symmetry and the Casimir effect," Nuclear Physics B, vol. 217, no. 2, pp. 336-348, 1983.

[3] H. Aoyama, "Casimir energy of Higgs-field configurations in a Coleman-Weinberg-type theory," Physical Review D, vol. 29, no. 8, pp. 1763-1771, 1984.

[4] Y. Igarashi, "Supersymmetry and the Casimir effect between plates," Physical Review. D, vol. 30, no. 8, pp. 1812-1820, 1984.

[5] T. Inami and K. Yamagishi, "Vanishing quantum vacuum energy in leven-dimensional supergravity on the round seven-sphere," Physics Letters B, vol. 143, no. 1-3, pp. 115-120, 1984.

[6] K. Kikkawa, T. Kubota, S. Sawada, and M. Yamasaki, "Stability of self-consistent dimensional reduction," Physics Letters B, vol. 144, no. 5-6, pp. 365-369, 1984.

[7] K. Kikkawa and M. Yamasaki, "Casimir effects in superstring theories," Physics Letters B, vol. 149, no. 4-5, pp. 357-360, 1984. 
[8] K. A. Milton and Y. J. Ng, "Maxwell-chern-simons Casimir effect," Physical Review D, vol. 42, no. 8, pp. 2875-2880, 1990.

[9] I. Brevik and R. Sollie, "On the Casimir energy for a $2 N$-piece relativistic string," Journal of Mathematical Physics, vol. 38, no. 6, pp. 2774-2785, 1997.

[10] M. Fabinger and P. Hořava, "Casimir effect between world-branes in heterotic M-theory," Nuclear Physics. B, vol. 580, no. 1-2, pp. 243-263, 2000.

[11] S. Nojiri, S. D. Odintsov, and S. Zerbini, "Bulk versus boundary (gravitational Casimir) effects in the quantum creation of an inflationary brane-world universe," Classical and Quantum Gravity, vol. 17, no. 23, pp. 4855-4866, 2000.

[12] M. Demetrian, "Casimir effect in four simple situations-including a noncommutative two-sphere," Fizika B, vol. 11, pp. 175-190, 2002.

[13] G. J. Maclay, H. Fearn, and P. W. Milonni, “Of some theoretical significance: implications of Casimir effects," European Journal of Physics, vol. 22, no. 4, pp. 463-469, 2001.

[14] M. S. R. Miltão, Estudo do Efeito Casimir Eletromagnético Esférico pelo Método da Dupla Regularização, Tese de Doutorado, Universidade Federal do Rio de Janeiro, 2004.

[15] G. Plunien, B. Müller, and W. Greiner, "The Casimir effect," Physics Reports, vol. 134, no. 2-3, pp. 87-193, 1986.

[16] S. K. Lamoreaux, "Resource letter CF-1: Casimir force," American Journal of Physics, vol. 67, no. 10, pp. 850-861, 1998.

[17] M. Bordag, U. Mohideen, and V. M. Mostepanenko, "New developments in the Casimir effect," Physics Reports, vol. 353, no. 1-3, pp. 1-205, 2001.

[18] P. W. Milonni, The Quantum Vacuum: An Introduction to Quantum Electrodynamics, Academic Press, Boston, Mass, USA, 1994.

[19] E. Elizalde, S. D. Odintsov, A. Romeo, A. A. Bytsenko, and S. Zerbini, Zeta Regularization Techniques with Applications, World Scientific Publishing, River Edge, NJ, USA, 1994.

[20] E. Elizalde, Ten Physical Applications of Spectral Zeta Functions, Springer, Berlin, Germany, 1995.

[21] V. M. Mostepanenko and N. N. Trunov, The Casimir Effect and Its Applications, Clarendon Press, Oxford, UK, 1997.

[22] K. A. Milton, The Casimir Effect, World Scientific Publishing, River Edge, NJ, USA, 2001.

[23] M. Bordag, G. L. Klimchitskaya, U. Mohideen, and V. M. Mostepanenko, Advances in the Casimir Effect, Oxford University Press, New York, NY, USA, 2009.

[24] B. Davies, "Quantum electromagnetic zero-point energy of a conducting spherical shell," Journal of Mathematical Physics, vol. 13, p. 1324, 1972.

[25] T. H. Boyer, "Quantum electromagnetic zero-point energy of a conducting spherical shell and the casimir model for a charged particle," Physical Review, vol. 174, no. 5, pp. 1764-1776, 1968.

[26] W. Lukosz, "Electromagnetic zero-point energy shift induced by conducting closed surfaces," Zeitschrift für Physik, vol. 258, no. 2, pp. 99-107, 1973.

[27] W. Lukosz, "Electromagnetic zero-point energy shift induced by conducting surfaces-II. The infinite wedge and the rectangular cavity," Zeitschrift für Physik, vol. 262, no. 4, pp. 327-348, 1973.

[28] C. M. Bender and P. Hays, "Zero-point energy of fields in a finite volume," Physical Review D, vol. 14, no. 10 , pp. 2622-2632, 1976.

[29] Siddhartha Sen, "A calculation of the Casimir force on a circular boundary," Journal of Mathematical Physics, vol. 22, no. 12, pp. 2968-2973, 1981.

[30] J. Baacke and Y. Igarashi, "Casimir energy of confined massive quarks," Physical Review D, vol. 27, no. 2, pp. 460-463, 1983.

[31] R. Balian and B. Duplantier, "Electromagnetic waves near perfect conductors. II. Casimir effect," Annals of Physics, vol. 112, no. 1, pp. 165-208, 1978.

[32] K. Olaussen and F. Ravndal, "Electromagnetic vacuum fields in a spherical cavity," Nuclear Physics, Section B, vol. 192, no. 1, pp. 237-258, 1981.

[33] K. Olaussen and F. Ravndal, "Chromomagnetic vacuum fields in a spherical bag," Physics Letters B, vol. 100, no. 6, pp. 497-499, 1981.

[34] J. R. Ruggiero, A. H. Zimerman, and A. Villani, “Application of analitical regularization to the Casimir forces," Revista Brasileira de Física, vol. 7, p. 663, 1977.

[35] S. W. Hawking, "Zeta function regularization of path integrals in curved spacetime," Communications in Mathematical Physics, vol. 55, no. 2, pp. 133-148, 1977.

[36] M. Bordag, E. Elizalde, and K. Kirsten, "Heat kernel coefficients of the Laplace operator on the Ddimensional ball," Journal of Mathematical Physics, vol. 37, no. 2, pp. 895-916, 1996. 
[37] S. Leseduarte and August Romeo, "Complete zeta-function approach to the electromagnetic Casimir effect for spheres and circles," Annals of Physics, vol. 250, no. 2, pp. 448-484, 1996.

[38] N. Shtykovt and D. V. Vassilevich, "The heat kernel for deformed spheres," Journal of Physics A, vol. 28, no. 1, pp. L37-L43, 1995.

[39] A. A. Actor and I. Bender, "Boundaries immersed in a scalar quantum field," Fortschritte der Physik. Progress of Physics, vol. 44, no. 4, pp. 281-322, 1996.

[40] R. B. Rodrigues, N. F. Svaiter, and R. M. D. de Paola, "Vacuum stress tensor of a scalar field in a rectangular waveguide," Tech. Rep. CBPF-NF-015/01, 2001.

[41] A. A. Saharian, "The generalized Abel-Plana formula. Applications to Bessel functions and Casimir effect," Tech. Rep. IC/2000/14, 2000.

[42] S. A. Fulling and P. C. W. Davies, "Radiation from a moving mirror in two dimensional space-time: conformal anomaly," Proceedings of the Royal Society A, vol. 348, no. 1654, pp. 393-414, 1976.

[43] P. C. W. Davies, S. A. Fulling, and W. G. Unruh, "Energy-momentum tensor near an evaporating black hole," Physical Review D, vol. 13, no. 10, pp. 2720-2723, 1976.

[44] P. C.W. Davies and S. A. Fulling, "Quantum vacuum energy in two dimensional space-times," Proceedings of the Royal Society A, vol. 354, no. 1676, pp. 59-77, 1977.

[45] L. S. Brown and G. J. Maclay, "Vacuum stress between conducting plates: an image solution," Physical Review, vol. 184, no. 5, pp. 1272-1279, 1969.

[46] S. M. Christensen, "Vacuum expectation value of the stress tensor in an arbitrary curved background: the covariant point-separation method," Physical Review. D, vol. 14, no. 10, pp. 2490-2501, 1976.

[47] D. Deutsch and P. Candelas, "Boundary effects in quantum field theory," Physical Review. D, vol. 20, no. 12, pp. 3063-3080, 1979.

[48] P. Candelas, "Vacuum energy in the presence of dielectric and conducting surfaces," Annals of Physics, vol. 143, no. 2, pp. 241-295, 1982.

[49] J. Schwinger, L. L. de Raad Jr., and K. A. Milton, "Casimir effect in dielectrics," Annals of Physics, vol. 115 , no. 1 , pp. $1-23,1978$.

[50] J. Schwinger, "Casimir Effect in source theory," Letters in Mathematical Physics, vol. 1, no. 1, pp. 43-47, 1975.

[51] K. A. Milton, L. L. de Raad Jr., and J. Schwinger, "Casimir self-stress on a perfectly conducting spherical shell," Annals of Physics, vol. 115, no. 2, pp. 388-403, 1978.

[52] J. S. Dowker and R. Critchley, "Scalar effective Lagrangian in de Sitter space," Physical Review. D, vol. 13, no. 2, pp. 224-234, 1976.

[53] J. S. Høye, I. Brevik, and J. B. Aarseth, "Casimir problem of spherical dielectrics: quantum statistical and field theoretical approaches," Physical Review E, vol. 63, Article ID 51101, pp. 1-12, 2001.

[54] I. Klich, "Photon Green's function and Casimir energy in a medium," Physical Review D, vol. 64, no. 4, Article ID 045001, 2001.

[55] M. S. R. Miltão, M. V. Cougo-Pinto, and P. A. Maia-Neto, "Global approach with cut-off exponential function to spherical Casimir effect," Nuovo Cimento della Societa Italiana di Fisica B, vol. 121, no. 4, pp. 419-428, 2006.

[56] M. S.R. Miltão, "Casimir energy for a double spherical shell: a global mode sum approach," Physical Review D, vol. 78, no. 6, Article ID 065023, 10 pages, 2008.

[57] F. Pascoal, L. C. Céleri, S. S. Mizrahi, and M. H. Y. Moussa, "Dynamical Casimir effect for a massless scalar field between two concentric spherical shells," Physical Review A, vol. 78, no. 3, Article ID 032521, 2008.

[58] L. P. Teo, "Finite temperature Casimir effect for scalar field with Robin boundary conditions in spacetime with extra dimensions," Journal of High Energy Physics, vol. 2009, no. 11, article 095, p. 95, 2009.

[59] C. C. Ttira and C. D. Fosco, "Casimir effect at finite temperature in a real scalar field theory," http://arxiv.org/abs/0809.2589.

[60] G. M. Napolitano, G. Esposito, and L. Rosa, “Energy-momentum tensor for a scalar Casimir apparatus in a weak gravitational field: neumann conditions," Physical Review D, vol. 78, no. 10, 2008.

[61] G. Esposito, G. M. Napolitano, and L. Rosa, “Energy-momentum tensor of a Casimir apparatus in a weak gravitational field: scalar case," Physical Review D, vol. 77, no. 10, Article ID 105011, p. 7, 2008.

[62] P. Moylan, "The casimir effect for a massless scalar field in the $\mathrm{n}$ dimensional Einstein universe with Dirichlet boundary conditions on a sphere," Journal of Physics: Conference Series, vol. 128, Article ID 012010, 2008.

[63] R. Linares, H. A. Morales-Técotl, and O. Pedraza, “Casimir force for a scalar field in warped brane worlds," Physical Review D, vol. 77, no. 6, Article ID 066012, 2008. 
[64] T. Emig and R. L. Jaffe, "Casimir forces between arbitrary compact objects," Journal of Physics. A, vol. 41, no. 16, Article ID 164001, 21 pages, 2008.

[65] L. V. Ahlfors, Complex Analysis, McGraw-Hill, Auckland, New Zealand, 3rd edition, 1978.

[66] A. Lins Neto, "Funções de uma variável complexa," Projeto Euclides, IMPA, Rio de Janeiro, Brazil, 1996.

[67] M. R. Spiegel, Complex Variables, McGraw-Hill, New York, NY, USA, 1997.

[68] M. E. Bowers and C. R. Hagen, "Casimir energy of a spherical shell," Physical Review. D, vol. 59, no. 2, Article ID 025007, 6 pages, 1999.

[69] T. H. Boyer, "Quantum zero-point energy and long-range forces," Annals of Physics, vol. 56, pp. 474503, 1970.

[70] T. H. Boyer, "Quantum electromagnetic zero-point energy of a conducting spherical shell and the casimir model for a charged particle," Physical Review, vol. 174, no. 5, pp. 1764-1776, 1968.

[71] G. N. Watson, A Treatise on the Theory of Bessel Functions, Cambridge University Press, Cambridge, UK, 1966.

[72] M. Abramovitz and I. A. Stegun, Handbook of Mathematical Functions, Dover, New York, NY, USA, 1972.

[73] K. Knopp, Theory and Application of Infinity Series, Blackie and Son, London, UK, 1964.

[74] G. Barton, "On the finite difference between divergent sum and integral," Journal of Physics A: General Physics, vol. 14, no. 5, article 021, pp. 1009-1027, 1981.

[75] G. Barton, "Addendum to 'On the finite difference between divergent sum and integral', "Journal of Physics A: Mathematical and General, vol. 15, no. 1, pp. 323-326, 1982.

[76] I. Brevik and H. Kolbenstvedt, "Electromagnetic Casimir densities in dielectric spherical media," Annals of Physics, vol. 149, no. 2, pp. 237-253, 1983.

[77] C. M. Bender and K. A. Milton, "Scalar Casimir effect for a D-dimensional sphere," Physical Review D, vol. 50, no. 10, pp. 6547-6555, 1994.

[78] S. A. Fulling, "Systematics of the relationship between vacuum energy calculations and heat-kernel coefficients," Journal of Physics. A. Mathematical and General, vol. 36, no. 24, pp. 6857-6873, 2003.

[79] K. A. Milton, "Calculating Casimir energies in renormalizable quantum field theory," Physical Review D, vol. 68, no. 6, Article ID 065020, 2003.

[80] Kimball A. Milton, "Casimir energies and pressures for $\delta$-function potentials," Journal of Physics. A. Mathematical and General, vol. 37, no. 24, pp. 6391-6406, 2004.

[81] K. A. Milton, "The Casimir effect: recent controversies and progress," Journal of Physics. A, vol. 37, no. 38, pp. R209-R277, 2004. 

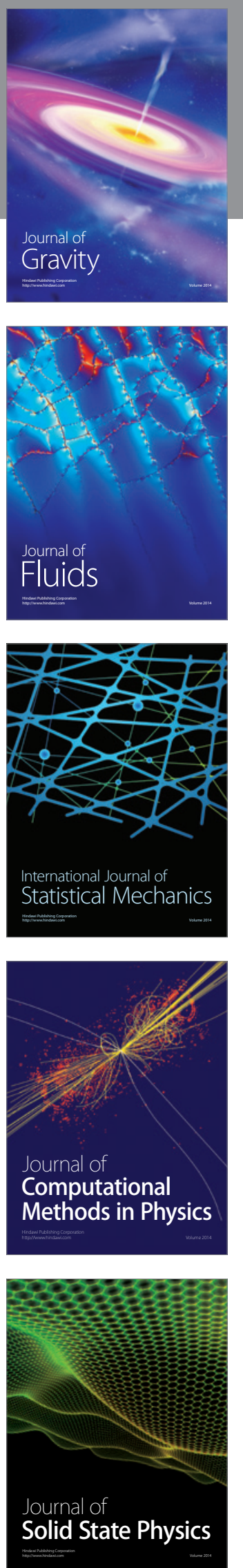

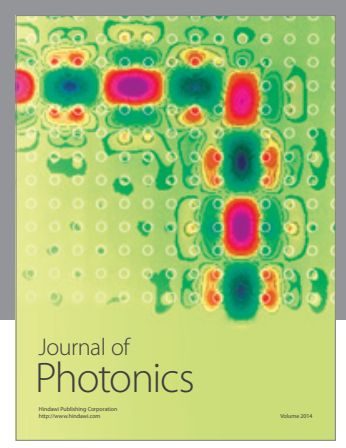

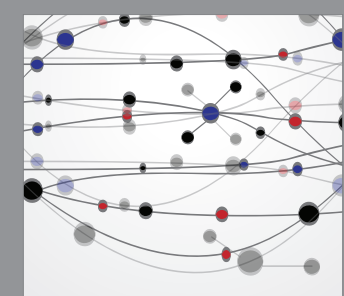

The Scientific World Journal
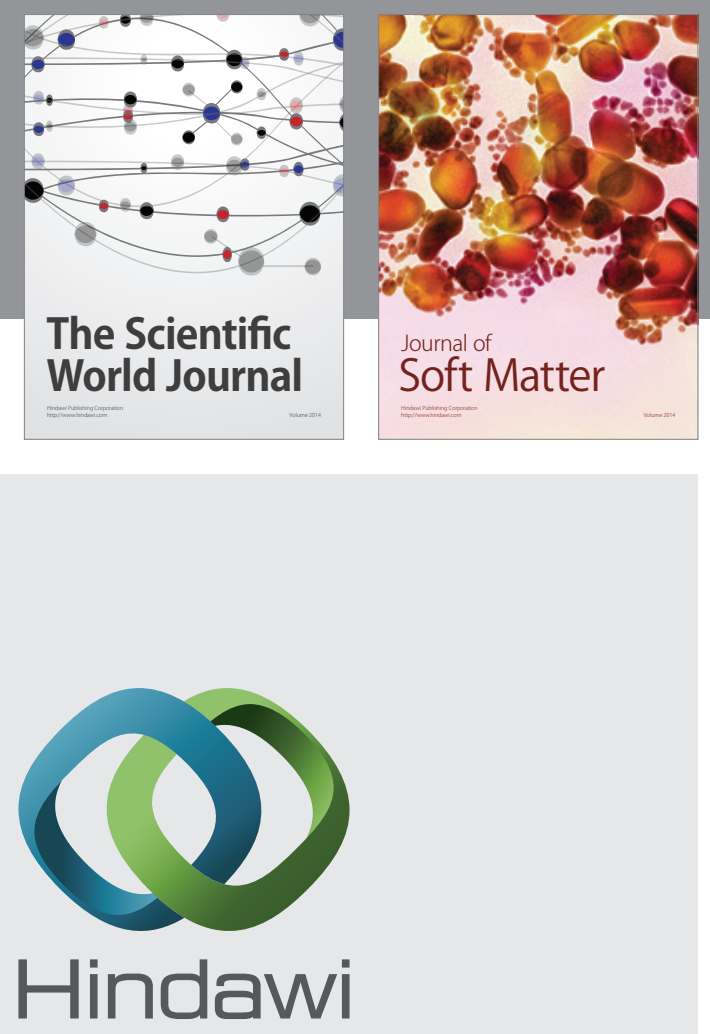

Submit your manuscripts at

http://www.hindawi.com
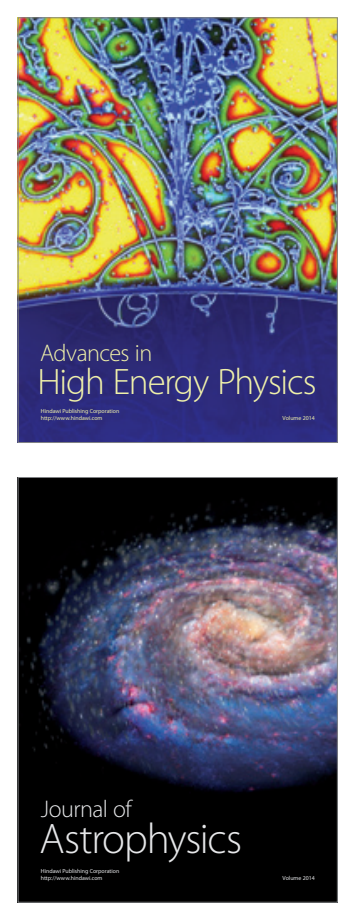
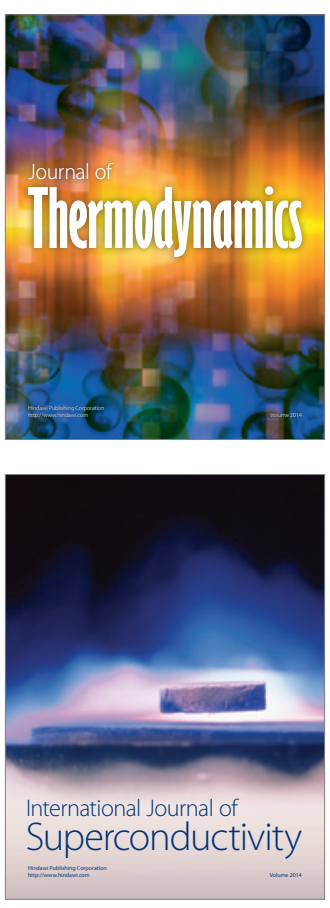
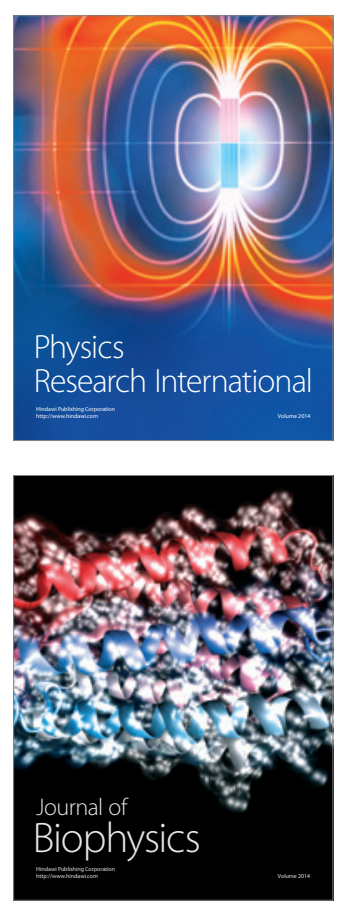
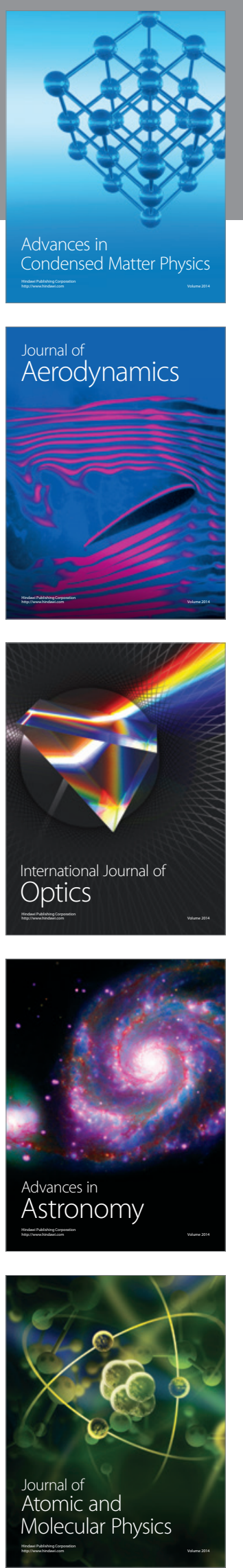